\title{
Clinical and Epidemiological aspects of SARS-CoV-2 infection among pregnant and postpartum women in Mozambique: a prospective cohort study
}

Charles M'poca Charles ( $\nabla$ cmpoca@gmail.com )

University of Campinas https://orcid.org/0000-0001-7133-0574

Nafissa Bique Osman

Department of Obstetric and Gynecology, Maputo Central Hospital, Mozambique

\section{Domingos Arijama}

Department of Obstetric and Gynecology, maputo central Hospital, Maputo, Mozambique.

\section{Benjamim Matingane}

Department of Obstetric and Gynecology, Maputo Central Hospital, Maputo, Mozambique

\section{Tomás Sitoé}

Department of Obstetric and Gynecology, Maputo Central Hospital, Maputo, Mozambique.

\section{Darlene Kenga}

Department of Microbiology, Faculty of Medicine, Eduardo Mondlane University, Maputo, Mozambique.

\section{Cesaltina Lorenzoni}

Pathology Department, Faculty of Medicine, Eduardo Mondlane University, Maputo, Mozambique.

\section{Elvira Luís}

Department of Obstetric and Gynecology, Maputo Central Hospital, Maputo, Mozambique.

\section{Rodolfo Pacagnella}

Department of Obstetric and Gynecology, School of Medicine, University of Campinas, Sao paulo, Brazil.

\section{Jahit Sacarlal}

Department of Microbiology, Faculty of Medicine, Eduardo Mondlane University, Maputo, Mozambique.

\section{Research}

Keywords: COVID-19, risk factors, maternal and perinatal morbidity, low-income country, Mozambique

Posted Date: September 29th, 2021

DOI: https://doi.org/10.21203/rs.3.rs-936594/v1

License: (c) (i) This work is licensed under a Creative Commons Attribution 4.0 International License. Read Full License 


\section{Abstract \\ Background}

Although there is a significant increase of evidence regarding the prevalence and impact of COVID-19 on maternal and perinatal outcomes, data on the effects of the pandemic on the obstetric population in sub-Saharan African countries are still scarce. Therefore, the study aims were to assess the prevalence and impact of COVID-19 on maternal and neonatal outcomes in the obstetric population at Central Hospital of Maputo (HCM), Mozambique.

\section{Methods}

Prospective cohort study conducted at teaching and referral maternity, HCM, from 20 October 2020 to 22 July 2021. We collected maternal and perinatal outcomes up to six weeks postpartum of eligible women (pregnant and postpartum women - up to the 14th day postpartum) screened for COVID-19 (individual test for symptomatic participants and pool testing for asymptomatic). The primary outcome was maternal death, SARS and UCI admission. We estimated the COVID-19 prevalence and the unadjusted RR $(95 \% \mathrm{CI})$ for maternal and perinatal outcomes. We used the chi-square or Fisher's exact test to compare qualitative variables (two-sided $p$-value $<0.05$ for statistical significance).

\section{Results}

We included 239 participants. The overall prevalence of COVID-19 was 9.2\% (22/239) and in the symptomatic group was $32.4 \%(11 / 34)$. About $48 \%$ of the participants with COVID-19 were asymptomatic. Moreover, the most frequent symptoms were dyspnoea (33.3\%), cough (28.6\%), anosmia (23.8\%), and fever (19\%). Not having a partner, being pregnant, and consuming alcohol were vulnerability factors for SARS-CoV-2 infection. COVID-19 among pregnant and postpartum women was associated with an increased risk of adverse maternal outcome, foetal death $(\mathrm{RR}=4.0$ [1.19-13.48]) and abortion/stillbirth 12.0 [7.7-18.7]). Conversely, we did not observe a significant difference in the primary outcomes between exposed and non-exposed groups.

\section{Conclusion}

The prevalence of COVID-19 in the obstetric population is higher than in the general population, and most pregnant and postpartum women with COVID-19 infection are asymptomatic. Being pregnant, not having a partner and alcohol consumption were factors of greatest vulnerability to SARS-COV-2 infection. Data suggest that pregnant women with COVID-19 may have a higher risk of adverse gestational outcomes, reinforcing the need for universal testing and monitoring of this population group. However, more prospective and robust studies are needed to assess these outcomes.

\section{Plain English Summary}

The epidemiological pattern of the COVID-19 pandemic in Africa is heterogeneous, and many African countries are still struggling to establish efficient testing policy, guarantee sufficient laboratory supply and achieve or maintain adequate testing capacity. In addition, evidence suggests that sexual and reproductive health services were the 
most affected by the pandemic; this scenario might have devastating effects on maternal and perinatal health. Moreover, the SARS-CoV-2 infection among pregnant and postpartum women is associated with an increased risk of adverse maternal and neonatal health (preterm birth, preeclampsia and maternal death).

Although there is a significant increase of evidence regarding the prevalence and impact of COVID-19 on maternal and perinatal health, data on the effects of this condition on the obstetric population in low-income countries are scarce. Therefore, the study aims were to assess the prevalence and impact of COVID-19 on maternal and neonatal health at referral maternity in Maputo, Mozambique.

Our findings suggest that the prevalence of COVID-19 in the obstetric population is higher than the general population, and most pregnant and postpartum women are asymptomatic. Being pregnant, not having a partner and alcohol consumption were factors of greatest vulnerability to SARS-COV-2 infection. Moreover, our pregnant and postpartum women with COVID-19 may have a higher risk of adverse gestational outcomes (foetal death and abortion). Therefore, universal screening might be the best approach to be implemented where resources are available to ensure adequate management of pregnant women and newborn.

\section{Background}

On the African continent, contrarily to previously developed prediction models, epidemiological data suggest that the progression of the first and second wave of the pandemic was slower, with fewer reported cases and lower disease-related mortality rate (1-3). However, the epidemiological pattern of the COVID-19 pandemic in Africa is quite heterogeneous: about four-fifths (82.6\%) of cases were reported in 9 of the 55 countries of the African Union (4). This heterogeneous pattern is due to several factors as the young population, low testing capacity, weak and inefficient epidemiological surveillance systems (4).

Many African countries are still struggling to establish efficient testing policy, guarantee sufficient laboratory supply and achieve or maintain the adequate testing capacity, testing at the level of ten negative tests to one positive (test per case ratio $\geq 10)(5,6)$. Nevertheless, during the pandemic, in most African countries, the COVID-19 diagnostic capacity was expanded through the GeneXpert platforms previously deployed to diagnose tuberculosis $(7,8)$, and Mozambique was not an exception.

The first case of COVID-19 was reported on 22 March 2020. As of 19 September 2021, the Mozambique Ministry of Health (MoH) had reported 150018 cases (tests per case ratio: 5.9) and 1903 COVID-19 deaths (case fatality ratio: $1.27 \%$ ) (9). Data suggest that sexual and reproductive health (SSR) services were the most affected by the pandemic, reducing or interrupting these services in more than $50 \%$ of cases (10). Furthermore, this reduction in the provision of services might have devastating effects on maternal and perinatal health due to the increase in maternal and child mortality (11).

The prevalence of COVID-19 in pregnancy was estimated at $41 \%$ in symptom-based screening (12) or $7 \%$ in universal screening (13). However, the COVID-19 prevalence can vary according to several factors, for example, epidemiological patterns of COVID-19 in the region and country, type of test used for SARS-CoV-2 detection and testing policy (universal or symptoms based screening), among others.

The SARS-CoV-2 infection among pregnant and postpartum women is associated with an increased risk of adverse maternal and perinatal outcomes $(14,15)$. In addition, data suggest that pregnant women with advanced age, black race, obesity, and associated comorbidities such as hypertension, and diabetes mellitus have a higher risk of 
severity $(15,16)$. These maternal and perinatal health effects are disproportionately higher in the low-income population, where health systems are fragile and less responsive to extreme adverse public health events (17). For example, in Mozambique, hospitals are not adequately equipped ( $0.4 \%$ of hospitals with oxygen therapy available) and have low geographic accessibility (18).

Although there is a significant increase of evidence regarding the prevalence and impact of COVID-19 on maternal and perinatal outcomes (19), data on the effects of the pandemic on the obstetric population in sub-Saharan African countries are still scarce. Therefore, the present study aims to assess the prevalence and impact of COVID19 on maternal and neonatal outcomes in the obstetric population admitted to the maternity hospital of the Central Hospital of Maputo (HCM), Mozambique.

\section{Methods}

\section{Study population and study location}

A prospective cohort study included pregnant and postpartum women (up to the 14th day of postpartum), asymptomatic or diagnosed with flu syndrome and / or suspected COVID-19, regardless of age, admitted to the Gynaecology and Obstetrics service of the Central Hospital of Maputo (HCM), Mozambique, from 20 October 2020 to 22 July 2021.

The HCM is a teaching and referral maternity hospital for the region and the country, with comprehensive obstetric care. The screening for SARS-CoV-19 infection in pregnant and postpartum women is similar to the general population, focused on symptomatic individuals or those with a history of contact with a positive case. We intentionally estimated a sample size of 300 participants (pairs of pregnant women and newborn) as the evidence on the effects of COVID-19 on pregnancy was paucity when we were implementing our study.

\section{Procedure}

The study protocol included pregnant (regardless of the gestational age) and postpartum women (up to 14th day of puerperium) who attended the HCM obstetrical and gynaecological services and provided or signed the consent form. At hospital admission or soon after, the research team (nurses, resident doctors and consultant obstetrician) identified, invited and assessed for eligibility criteria all potentials participants (in the emergency room and/or patient wards) after giving complete study information, including procedures.

After reading and signing the informed consent form, the participants were asked to provide upper respiratory specimens for laboratory screening of SARS-CoV-2 infection. We excluded all women with invalid telephone numbers who did not accept providing upper respiratory specimens or withdrew their consent form during the study.

We collected nasopharyngeal and oropharyngeal specimens through swabs. For asymptomatic patients, we collected specimens in duplicate. After collecting the specimens, they were placed in a viral transport medium (VTM) containing antifungal and antibiotic supplements. We storage and shipped the specimens in cooler boxes on ice (at $\left.2-8^{\circ} \mathrm{C}\right)$ to the local laboratory for viral detection. All sample viral detection was done via GeneXpert platforms for COVID-19, and the results were available within 24 hours (2hour for symptomatic participants and 24 hours for asymptomatic participants). 
The laboratory detention virus followed two approaches: the symptomatic participants and/or severe acute respiratory illness and high-risk contacts were individually tested. Conversely, samples from asymptomatic participants with no history of positive contact for COVID-19 were tested using a pool testing strategy. Pool testing is a technique in which specimens collected from different participants are organised into groups ('pools') and tested together (20). At the time of study implementation, the data from the Mozambican obstetric population suggested that the prevalence of COVID-19 was around 6\% (21). Therefore, we estimated a pool of nine samples (P9S3) analysed in three stages $(20,22)$. The pool tests positive was further divided into sub-pools of three specimens before retesting each specimen in the pool individually to determine which individual(s) are positive.

The specimens' collection, processing, and testing were carried out by health professionals previously trained for this purpose and according to the standards recommended by the Ministry of Health Mozambique and the World Health Organization for collecting and handling clinical specimens for COVID-19 testing.

Subsequently, the included participants were allocated into two groups according to the test result. The exposed group consisted of pregnant and postpartum women (up to the 14th day) with a positive test for SARS-CoV-2 infection. The non-exposed group consisted of pregnant and postpartum women with a negative test. During the follow-up, participants with a negative test could move to the exposed group if they were positive for SARS-CoV-2 infection COVID-19 when retested.

During inclusion and follow-up (until the 6th week postpartum), data on sociodemographic characteristics, clinical characteristics of SARS-CoV-2 infection, adverse maternal events and maternal and gestational outcomes were collected. Study data were collected and managed using REDCap (Research Electronic Data Capture) electronic data capture tools installed in smartphones (tablets) hosted at Eduardo Mondlane University, Maputo, Mozambique $(23,24)$.

The primary outcome was the severe maternal outcome (maternal death, SARS and UCI admission). Secondary outcomes were: pregnancy outcomes (abortion, foetal death), preterm birth, preeclampsia/ eclampsia, mode of delivery, Apgar, NICU admission, neonatal death, congenital anomaly and any composite of adverse pregnancy outcome (NICU admission, preterm birth, foetal death, neonatal death, miscarriage/abortion). In addition, we have considered potential confounders variables, other viral respiratory syndromes, history of adverse pregnancy outcomes, and all factors related to the three-delay model in obstetric care.

\section{Statistical analysis}

We describe and compare the sociodemographic, obstetric and clinical characteristics of pregnant and postpartum women included in the study according to exposure (group exposed and not exposed to COVID-19). Likewise, we estimated the prevalence of COVID-19 in the general population, in the symptomatic and asymptomatic groups, and compared the clinical and severity characteristics in the group of symptomatic women according to the exposure group and estimated the level of significance (we considered Two-sided p-value $<0.05$ as statistically significant).

We additionally have considered the time of symptom onset before admission, the duration of symptoms, the most prevalent symptoms, the type of management at the time of admission, admission to the intensive care unit and the presence of the severe acute respiratory syndrome. Finally, we estimated the unadjusted relative risk with a $95 \%$ interval to evaluate the risk of adverse maternal and perinatal outcomes. For comparisons of qualitative variables, 
we used the chi-square test or Fisher's exact test when indicated. Statistical analyses were performed using the

IBM SPSS statistic program (version 27.0).

\section{Ethical Issues}

The study protocol was approved by the Mozambique National Review Board (Letter of approval number 61/CNBS/2020). Moreover, all participants were fully informed regarding the study procedure and provided written or oral consent before their inclusion in the study. In addition, all participants had adequate clinical management (for SARS-CoV-2 positive cases) and psychological support when needed.

\section{Results}

We included 239 participants; 22 were exposed (COVID-19 confirmed), and 217 were not exposed (COVID-19 negative). Maternal and neonatal outcomes were available in $93 \%$ of the included participants (223/239) (Fig. 1). The average age was 28 years (SD 6.1), and the majority of the population was Black (92.1\% [220/239]).

At the time of study admission, about 37\% (83/226) were pregnant, two-thirds of the participants had had at least four antenatal care consultations, and the majority (69.4\% [150/216]) of the participants had prenatal consultations in public services (Table 1). 
Table 1

Sociodemographic and obstetric characteristics of Maputo Central Hospital included in the study $(n=239)$.

\begin{tabular}{|c|c|c|c|}
\hline Characteristics & $\begin{array}{l}\text { Confirmed COVID-19 } \\
n=22\end{array}$ & $\begin{array}{l}\text { Negative COVID-19 } \\
\mathrm{n}=217\end{array}$ & $p$-value \\
\hline $\operatorname{Age}^{a}$ & & & 0.326 \\
\hline$\leq 19$ & $2(9,1 \%)$ & $15(7,4 \%)$ & \\
\hline $20-35$ & $14(63,6 \%)$ & $155(76,0 \%)$ & \\
\hline$>35$ & $6(27,3 \%)$ & $34(16,7 \%)$ & \\
\hline Ethnicity ${ }^{b}$ & & & 0.390 \\
\hline Black & $20(95.2 \%)$ & $200(98.0 \%)$ & \\
\hline Non-black & $1(4.8 \%)$ & $4(2.0 \%)$ & \\
\hline Area of residence ${ }^{c}$ & & & 0.461 \\
\hline Peri-urban & $19(86.4 \%)$ & $183(90.6 \%)$ & \\
\hline Urban & $3(13.6 \%)$ & $19(9.4 \%)$ & \\
\hline Marital Status ${ }^{d}$ & & & 0.043 \\
\hline With partner & $13(61.9 \%)$ & $164(80.8 \%)$ & \\
\hline Without partner & $8(38.1 \%)$ & $39(19.2 \%)$ & \\
\hline Schooling ${ }^{\mathrm{e}}$ & & & 0.883 \\
\hline None or Primary or Secondary & $12(63.2 \%)$ & $131(64.9 \%)$ & \\
\hline College or more & $7(36.8 \%)$ & $71(35.1 \%)$ & \\
\hline Usual means of transport ${ }^{f}$ & & & 0.096 \\
\hline Public & $10(52.6 \%)$ & $140(71.1 \%)$ & \\
\hline Private & $9(47.4 \%)$ & $57(28.9 \%)$ & \\
\hline Source of antenatal care ${ }^{g}$ & & & 0.112 \\
\hline Public & $17(68.9 \%)$ & $181(94.3 \%)$ & \\
\hline Private & $3(15.0 \%)$ & $11(5.7 \%)$ & \\
\hline ANC consultation ${ }^{\mathrm{h}}$ & & & 0.667 \\
\hline None & $1(5.0 \%)$ & $7(3.5 \%)$ & \\
\hline $1-3$ & $5(25.0 \%)$ & $60(30.0 \%)$ & \\
\hline$\geq 4$ & $14(70.0 \%)$ & $133(66.5 \%)$ & \\
\hline
\end{tabular}




\begin{tabular}{|c|c|c|c|}
\hline Characteristics & $\begin{array}{l}\text { Confirmed COVID-19 } \\
n=22\end{array}$ & $\begin{array}{l}\text { Negative COVID-19 } \\
n=217\end{array}$ & $p$-value \\
\hline Provenience $^{i}$ & & & 1.00 \\
\hline Home & $18(81.8 \%)$ & $168(82.8 \%)$ & \\
\hline Referral & $4(18.2 \%)$ & $35(17.2 \%)$ & \\
\hline Parity j & & & 0.447 \\
\hline 0 & $12(57.1 \%)$ & $87(42.0 \%)$ & \\
\hline $1-2$ & $7(33.3 \%)$ & $98(47.3 \%)$ & \\
\hline$\geq 3$ & $2(9.5 \%)$ & $22(10.6 \%)$ & \\
\hline Planned pregnancy k & $11(52.4 \%)$ & $134(65.4 \%)$ & 0.237 \\
\hline Multiple pregnancy & $1(4.8 \%)$ & $7(3.4 \%)$ & 0.544 \\
\hline Pregnancy status at enrolment & & & 0.000 \\
\hline Pregnancy & $17(81.0 \%)$ & $66(32.2 \%)$ & \\
\hline Post-partum & $4(19.0 \%)$ & $139(67.8 \%)$ & \\
\hline Chronic hypertension & $1(4.5 \%)$ & $6(2.8 \%)$ & 0.496 \\
\hline Pre-existing diabetes & $0(0.0 \%)$ & $0(0.0 \%)$ & NA \\
\hline Asthma & $1(4.5 \%)$ & $2(0.9 \%)$ & 0.252 \\
\hline Anemia & $0(0.0 \%)$ & $10(4.6 \%)$ & 0.427 \\
\hline HIV & $2(9.1 \%)$ & $28(12.9 \%)$ & 0.749 \\
\hline Alcohol drinking & $13(65.0 \%)$ & $62(31.0 \%)$ & 0.002 \\
\hline Symptoms $^{\mathrm{m}}$ & & & 0.000 \\
\hline Yes & $11(52.4 \%)$ & $22(10.9 \%)$ & \\
\hline No & $10(47.6 \%)$ & $180(89.1)$ & \\
\hline
\end{tabular}

The overall prevalence of COVID-19 was 9.2\% (22/239) and in the symptomatic group was 32.4\% (11/34) (Fig. 2A and $2 \mathrm{~B}$ ). About $48 \%$ of the participants with COVID-19 were asymptomatic (Fig. 2C). Dyspnoea (33.3\%), cough (28.6\%), anosmia (23.8\%), and fever (19\%) were more frequent symptoms. Hyposmia/anosmia and ageusia were symptoms statistically associated with COVID-19 diagnoses (Table 1).

The sociodemographic factors significantly associated with increased risk of SARC-CoV-2 infection were not having a partner, being pregnant, and consuming alcohol during pregnancy (Table 1). There were non-significant differences between the exposed and non-exposed groups for the following variables: duration of symptoms, initial management, the presence of severe acute respiratory syndrome and admission to the intensive care unit at any 
time (Table 2). The diagnosis of COVID-19 among pregnant and postpartum women was associated with an increased risk of adverse maternal outcomes, foetal death $(R R=4.0$ [1.19-13.48]) and abortion/stillbirth (12.0 [7.7-18.7]) (Table 3). On the other hand, there were no statistically significant differences in the remain maternal and perinatal outcomes between exposed and non-exposed groups (Table 3). Moreover, during the cohort followup, we did not record any cases of maternal death.

Table 2

Clinical features and severity of COVID-19 infection among symptomatic women $(n=32)$.

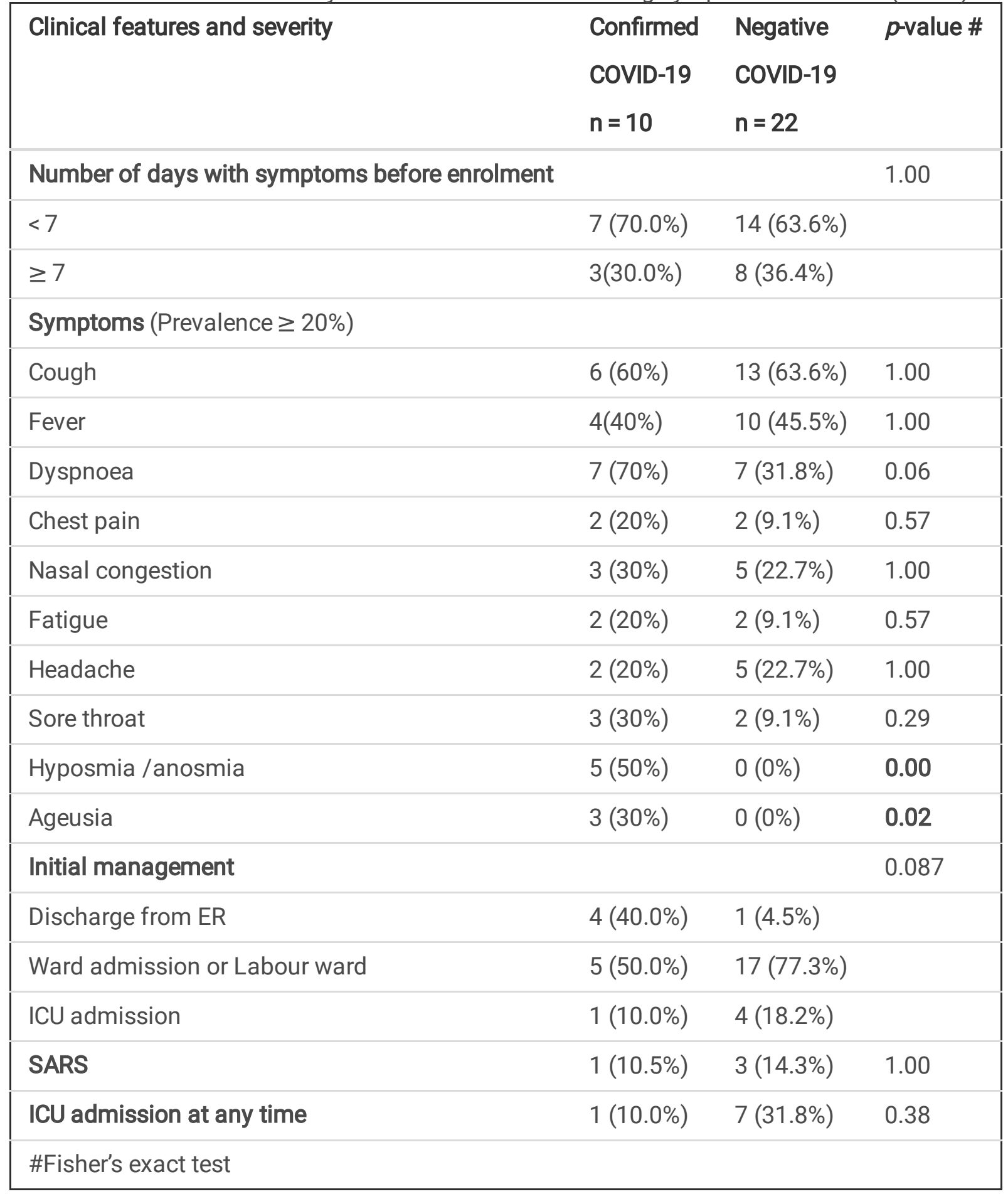


Table 3

Risk estimates for adverse pregnancy and neonatal outcomes according to COVID-19 exposure $(n=223)$.

\section{Pregnancy outcomes}

\section{COVID-19 positive}

$$
n=21
$$

\section{Pregnancy outcome}

Abortion

Foetal Death

Live birth

Preterm birth ${ }^{a}$

Pre-clampsia

Mode of delivery

Vaginal birth

Elective C-section

Intrapartum C-section

Apgar $<7$ at 1 rst minute ${ }^{b}$

Apgar $<7$ at 5th minute ${ }^{b}$

Neonatal respiratory distress ${ }^{c}$

Neonatal mechanical ventilation ${ }^{\mathrm{d}}$

NICU admission ${ }^{\mathrm{e}}$

Any neonatal morbidity

Congenital anomaly

Neonatal death

Any APO/WHO*

Any gestational intercurrence **

$1(4.8 \%)$

$2(9.5 \%)$

$18(85.7 \%)$

$3(15.0 \%)$

$1(4.8 \%)$

$18(85.7 \%)$

$2(9.5 \%)$

$1(4.8 \%)$

$1(5.9 \%)$

$0(0.0 \%)$

$0(0.0 \%)$

$0(0.0 \%)$

$2(12.5 \%)$

$2(9.5 \%)$

$0(0.0 \%)$

$0(0.0 \%)$

$7(33.3 \%)$

$5(23.8 \%)$
COVID-19 negative

$\mathrm{RR}$ [95\% Cl]

$\mathrm{n}=\mathbf{2 0 2}$
12.0 [7.7-18.7]

4.0 [1.19-13.48]

Ref.

$198(98.0 \%)$

49 (25.4\%)

$52(25.7 \%)$

$0.55[0.17-1.79]$

$0.14[0.02-1.10]$
$154(76.2 \%) \quad$ Ref.

$21(10.4 \%)$

$0.83[0.21-3.35]$

$27(13.4 \%)$

0.34 [0.05-2.46]

$36(19.1 \%)$

0.28 [0.04-2.07]

$14(7.4 \%)$

$25(14.7 \%)$

$8(4.7 \%)$

$36(20.6 \%)$

0.55 [0.12-2.54]

43 (21.3\%)

0.39 [0.09-1.73]

2 (1.12\%)

5 (2.9\%)

67(33.2\%)

1.01 [0.39-2.61]

48 (23.8\%)
1.00

Missing information a) 10, b) 18, c) 37, d) 35 , e) 25 .

* APO (any adverse pregnancy outcome): NICU admission, preterm birth, foetal death, neonatal death, miscarriage/abortion.

**hyperemesis, Foetal growth restriction, haemorrhage during pregnancy, threatened preterm labour.

\section{Discussion}

This prospective and exploratory study report the prevalence of COVID-19 in pregnancy and its impact on maternal and perinatal health in the obstetric population of Maputo, Mozambique. The overall prevalence of COVID-19 in pregnant and postpartum women was $9.2 \%$. Almost half of the population was asymptomatic at the time of 
diagnosis. In addition, the sociodemographic and gestational factors commonly associated with greater vulnerability to SARS-CoV-2 infection were being pregnant, alcohol consumption, and not having a partner.

These data suggest that the overall prevalence of COVID-19 in pregnant and postpartum women is higher than the general Mozambican population, which was 2-4\% (25). Likewise, this prevalence is relatively higher than that of the study in pregnant and postpartum women, also carried out in Maputo city (21). The difference in the COVID-19 prevalence might be due to the testing strategy, as the studies previously conducted in Mozambique (in general and obstetric population) were seroepidemiologic, and the COVID-19 pandemic magnitude in the country at the time of the studies implementation.

Conversely, our findings are similar to the results of the systematic review by Allotey and colleagues and another epidemiological study carried out in Zambia, which estimated an overall prevalence of COVID-19 in pregnant and postpartum women of $10 \%$ and $11.7 \%$, respectively $(15,26)$.

The prevalence of COVID-19 was $32.4 \%$ in the group of symptomatic women at study admission. These findings are similar to other studies in which testing was based on clinical symptoms $(15,27)$. Therefore, these data reinforce that the best testing approach is universal in places where resources are available to ensure proper management of pregnant women and newborns once even asymptomatic patients have an increased risk of maternal outcomes, maternal morbidity $(\mathrm{RR}, 1.24 ; 95 \% \mathrm{Cl}, 1.00-1.54)$ and preeclampsia $(\mathrm{RR}, 1.63 ; 95 \% \mathrm{Cl}, 1.01-2.63)$ (28).

Our data reinforce that SARS-CoV-2 infection is associated with an increased risk of adverse obstetric outcomes, such as foetal death. $(\mathrm{RR}=4.0$ [1.19-13.48]) and abortion/ stillbirth 12.0 [7.7-18.7]. These findings are similar to the systematic review, which estimated increased risk of stillbirth OR 1.29 (1.06-1.58)(14) and RR 2.84 (1.256.45)(15). The higher risk of adverse maternal outcomes observed in our cohort may be due to the third delay (receiving adequate and appropriate treatment)(29). Given that two-thirds of the participants in our study had at least four antenatal care, this delay might have been exacerbated by the COVID-19 pandemic.

We did not observe significant differences in the risk of admission to the intensive care unit, development of severe acute respiratory syndrome, preeclampsia, prematurity, NICU admission and neonatal death between the exposed and non-exposed groups. Our data are similar to systematic reviews $(14,30)$ and individual studies $(28)$. On the other hand, our findings differ from those of other published studies for maternal ICU admission outcomes, preeclampsia, which increased risk in pregnant women with COVID-19 $(30,31)$.

The major limitation of this study is related to the sample size. The sample size was small as it might not have the power to detect a difference between the exposure and non-exposure groups for some maternal and perinatal outcomes. Furthermore, although we have estimated a sample size of 300 participants (pairs of pregnant women and newborn), a scarcity of laboratory supplies (SARS-CoV-2 GeneXpert cartridges) at the national level hindered the study implementation. Therefore, reinforcing the difficulty of implementing prospective studies in places with few resources. In addition, the scarcity of SARS-CoV-2 GeneXpert cartridges might have influenced the lower test per COVID-19 case ratio de 5.8 observed in Mozambique, which is almost half of the recommended ratio.

The second limitation would be related to the testing strategy for the asymptomatic participant. Although the pooling test strategy might raise some concerns regarding the test performance (32), studies suggest that this testing modality could be implemented without compromising the sensitivity and specificity of the test $(20,33,34)$. 
We consider that this technique should be implemented in a low-resource setting (for example, Mozambique) to upscale the test capacity.

Another limitation would be the study setting. We implemented the study in a referral hospital with comprehensive and specialised obstetric care. In addition, we included a population mainly from the urban region; thus, the sample might not represent the entire population. Therefore, the study finding should be interpreted with caution, limiting their generalizability.

Conversely, our study has some strengths. First, we conducted a prospective study. Prospectively collected data were used to implement an adequate measure and appropriate COVID-19 cases management at the hospital level, with early isolation of positive cases, rational use of protective equipment and reduction of COVID-19 hospital transmission. Second, to the best of our knowledge, this is one of the first works developed in low-resource countries in sub-Saharan Africa and might be used as a baseline for future studies. Third, our study highlighted the role of modifiable factors (alcohol consumption) in the risk of SARS-CoV-2 infection. Likewise, the evidence of a risk increase in adverse gestational outcomes can raise awareness for greater attention to this group of patients and guide the construction and implementation of public policies to deal with COVID-19 in the obstetric population at the local and regional level.

\section{Conclusions}

The prevalence of COVID-19 in the obstetric population is higher than in the general population, and most pregnant and postpartum women with COVID-19 infection are asymptomatic. Being pregnant, not having a partner and alcohol consumption were factors of greatest vulnerability to SARS-COV-2 infection. Data suggest that pregnant women with COVID-19 may have a higher risk of adverse gestational outcomes, reinforcing the need for universal testing and monitoring of this population group. However, more prospective and robust studies are needed to assess these outcomes.

\section{Abbreviations}

ICF

Informed Consent Form

$\mathrm{HCM}$

Maputo Central Hospital

$\mathrm{MoH}$

Mozambique Ministry of Health

NICU

Neonatal Intensive Care Unit

P9S3

Pool of nine samples analysed in three stages

REDCap

Research Electronic Data Capture

$\mathrm{SRH}$

Sexual and Reproductive health

VTM

viral transport medium 


\section{Declarations}

\section{Ethics approval and consent to participate}

The study protocol was approved by the Mozambique National Review Board (Letter of approval number 61/CNBS/2020). Moreover, all participants were fully informed regarding the study procedure and provided written or oral consent before their inclusion in the study. In addition, all participants had adequate clinical management (for positive cases) and psychological support when needed.

\section{Availability of data and materials}

The datasets used during the current study are available from the corresponding author on reasonable request.

\section{Competing interests}

The authors declare that they have no competing interests.

\section{Funding}

This study received financial support from the National Research Fund (FNI) - Ministry of Science and Technology, Higher Education, Mozambique (Fundo Nacional de Investigação [FNI] - Ministério da Ciência e Tecnologia, Ensino Superior, Moçambique). Moreover, CMC received funding from SRH, part of the UNDP-UNFPA-UNICEF-WHO-World Bank Special Programme of Research, Development and Research Training in Human Reproduction (HRP), a cosponsored programme executed by the World Health Organisation (WHO), to complete his Post-graduate studies. However, the study funders had no role in the study design, data collection, analysis, interpretation, report writing, or decision to submit the manuscript. Therefore, this article represents the views of the named authors only and does not represent the views of the mentioned organisations.

\section{Authors' contributions}

CMC, RCP, NO, and JS conceived and designed the study. CMC, DK, BM, DA, TS, CL collected data. CMC, RCP, and EL were responsible for data analysis and interpretation. RCP and CMC wrote the first version of the manuscript. RCP, JS and NO critically reviewed the manuscript. All the authors read and approved the final version of the manuscript.

\section{Acknowledgements}

We would like to convey our gratitude to the remaining members of the Mozambique Study group of SARS-COV-2: Alfeu Passanduca, Alice Manjate, Aline Munezero, Cesaria Uassiquete, Filipe Majunta, Guilherme Morais Nobrega, Ilza Cambaza, José Carlos, José G Cecatti, Maria Laura Costa, Renato T Souza, Sérgio Taúnde and Tufária Mussá for their valuable suggestions and comments on the study design. Moreover, for their technical support and valuable contribution to the study implementation.

\section{References}

1. Massinga LM, Tshangela A, Salyer SJ, Varma JK, Ouma AEO, Nkengasong JN. COVID-19 in Africa: the spread and response. Nat Med. 2020;26(7):999-1003. 
2. Umviligihozo G, Mupfumi L, Sonela N, Naicker D, Obuku EA, Koofhethile C, et al. Sub-Saharan Africa preparedness and response to the COVID-19 pandemic: A perspective of early career African scientists. Wellcome Open Research 2020. 2020;5(163):163. 5(.

3. Walker PGT, Whittake C, Watson O, Marc B, Ainslie. Bhatia KEC S et al.

4. The Global Impact of COVID-19 and Strategies for Mitigation and Suppression. Imperial College London. (2020).2020. doi: https://doi.org/10.25561/77735.

5. Salyer SJ, Maeda J, Sembuche S, Kebede Y, Tshangela A, Moussif M, Ihekweazu C, Mayet N, Abate E, et al. The first and second waves of the COVID-19 pandemic in Africa: a cross- sectional study. Lancet. 2021;397:126575.

6. Organisation World Health Organisation. COVID-19 - virtual press conference - 30 March 2020: Geneve. 2020. [Available from: https://www.who.int/docs/default-source/coronaviruse/transcripts/who-audio-emergenciescoronavirus-press-conference-full-30mar2020.pdf?sfvrsn=6b68bc4a_2.

7. Ritchie H, Mathieu E, Rodés-Guirao L, Appel C, Giattino C, Ortiz-Ospina E, et al. Coronavirus Pandemic (COVID19). Our World in Data. 2021.

8. Cepheid Xpert Xpress SARS-CoV-2 has received FDA emergency use authorisation: Cepheid. 2020. [Available from: http://cepheid.mediaroom. com/2020-09-29-Cepheid-Receives-Emergency-Use-Authorization- For-SARSCoV-2-Flu-A-Flu-B-and-RSV-Combination-Test.

9. Chiang CY, Sony AE. Tackling the threat of COVID-19 in Africa: an urgent need for practical planning. Int J Tuberc Lung Dis. 2020, 24(5). doi:10.5588/ijtld.20.0192.

10. Ministério da Saúde, Direcção nacional de Saúde Pública, Centro Operativo de Emergência em Saúde Pública. Painel Epidemiológico SARS-CoV-2 - República de Moçambique: Maputo. 2021. [Available from: https://coesaude.maps.arcgis.com/apps/opsdashboard/index.html\#/8a0407dcf9374ae5a7b592e770c6d84f. (in portuguese).

11. World Health Organisation. Pulse survey on continuity of essential healt services during the COVID-19 pandemic: interim.

12. report, 27 August 2020. Geneva. 2020 [Available from: https://www.who.int/publications/i/item/WHO-2019nCoV-EHS_continuity-survey-2020.1.

13. Roberton T, Carter ED, Chou VB, Stegmuller AR, Jackson BD, Tam Y, et al. Early estimates of the indirect effects of the COVID-19 pandemic on maternal and child mortality in low-income and middle-income countries: a modelling study. The Lancet Global health. 2020;8(7).

14. Vivanti AJ, Mattern J, Vauloup-Fellous C, Jani J, Rigonnot L, Hachem LE, et al et al. Retrospective Description of Pregnant Women Infected with Severe Acute Respiratory Syndrome Coronavirus 2, France. Emerg Infect Dis. 2020. doi:10.3201/eid2609.202144.

15. Sakowicz A, Ayala AE, Ukeje CC, Witting CS, Grobman W, Miller Emily S. Risk factors for severe acute respiratory syndrome coronavirus 2 infection in pregnant women. Am J Obstet Gynecol MFM. 2020. doi:10.1016/j.ajogmf.2020.100198.

16. Chmielewska B, Barratt I, Townsend R, Kalafat E, Meulen J, Gurol-Urganci I, et al. Effects of the COVID-19 pandemic on maternal and perinatal outcomes: a systematic review and meta-analysis. Lancet Glob Health. 2021. doi:10.1016/S2214-109X(21)00079-6.

17. Allotey J, Stallings E, Bonet M, Yap M, Chatterjee S, Kew T, et al. Clinical manifestations, risk factors, and maternal and perinatal outcomes of coronavirus disease 2019 in pregnancy: living systematic review and 
meta-analysis. BMJ. 2020.

18. Takemoto M, Menezes MLS, Andreucci MO, Nakamura-Pereira CB, Amorim M, Katz MM. L, et al. The tragedy of COVID-19 in Brazil: 124 maternal deaths and counting. Int J Gynaecol Obstet. 2020. doi:10.1002/ijgo.13300. Epub 2020 Jul 29.

19. Charles CM, Amoah EM, Kourouma KR, et al. The SARS-CoV-2 pandemic scenario in Africa: What should be done to address the needs of pregnant women? Int J Gynaecol Obstet. 2020. doi:10.1002/ijgo.13403. Epub 2020 Oct 21.

20. Denhard KP, Chicumbe S, Muianga C, Laisse G, Aune K. Sheffel. How prepared is Mozambique to treat COVID19 patients? A new approach for estimating oxygen service availability, oxygen treatment capacity, and population access to oxygen-ready treatment facilities. Int J Equity Health. 2021.

DOI:https://doi.org/10.1186/s12939-021-01403-8.

21. Haghani M, Bliemer MCJ, Goerland Ft, Li J. The scientific literature on Coronaviruses, COVID-19 and its associated safety-related research dimensions: A scientometric analysis and scoping review. Saf sci. 2020. doi:10.1016/j.ssci.2020.104806. Epub 2020 May 7.

22. Eberhardt JN, Breuckmann NP, Eberhardt CS. Multi-Stage Group Testing Improves Efficiency of Large-Scale COVID-19 Screening. Journal of clinical virology: the official publication of the Pan American Society for Clinical Virology. 2020. doi: 10.1016/j.jcv.2020.104382. Epub 2020 Apr 23.

23. Mastala A, Neves A, Lumbandali N, Cumbane B, Matimbe R.

24. Massango A, et al. SARS-CoV-2 em Grávidas e Puérperas no Início da Transmissão.

25. Comunitária na Cidade de Maputo. Revista Moçambicana de Ciências de Saúde, vol.6, nº 1, 2020 | ISSN 2311-3308.(in portuguese).

26. Lohse S, Pfuhl T, Berkó-Göttel B, et al.Pooling of samples for testing for SARS-CoV-2 in asymptomatic people.Lancet Infect Dis. 2020; https://doi.org/10.1016/S1473-3099(20)30362-5.

27. Harris PA, Taylor R, Thielke R, Payne J, Gonzalez N, Conde JG. Research electronic data capture (REDCap) - A metadata-driven methodology and workflow process for providing translational research informatics support. J Biomed Inform. 2009 Apr;42(2):377-81.

28. Harris PA, Taylor R, Minor BL, Elliott V, Fernandez M, O’Neal L, et al.The REDCap consortium: Building an international community of software partners, J Biomed Inform. 2019 May 9 [doi: 10.1016/j.jbi.2019.103208].

29. Mozambique National Institute of Health. Most people exposed to COVID-19 have no symptoms 2020. August31. 2020. [Available from: https://covid19.ins.gov.mz/maior-parte-das-pessoas-expostas-a-covid-19nao-apresenta-sintomas/.(acessed 20 agust 2021, in portuguese).

30. Mulenga LB, Hines JZ, Fwoloshi S, Chirwa L, Siwingwa M, Yingst S, et al. Prevalence of SARS-CoV-2 in six districts in Zambia in July, 2020: a cross-sectional cluster sample survey. The Lancet Global health. 202.

31. Antoun L, Taweel NE, Ahmed I, Patni Si, Honest H. Maternal COVID-19 infection, clinical characteristics, pregnancy, and neonatal outcome: A prospective cohort study. Eur J Obstet Gynecol Reprod Biol. 2020. doi:10.1016/j.ejogrb.2020.07.008.

32. Villar J, Ariff S, Gunier RB, Thiruvengadam R, Rauch S, Kholin A, et al. Maternal and Neonatal Morbidity and Mortality Among Pregnant Women With and Without COVID-19 Infection: The INTERCOVID Multinational Cohort Study. JAMA pediatrics. 2021. doi:10.1001/jamapediatrics.2021.1050.

33. Thaddeus S, Maine S. D. Too far to walk: maternal mortality in context. S Soc Sci Med. 1994. doi:10.1016/0277-9536(94)90226-7. 
34. Huntley BJ, Mulder IA, Mascio DDi, Vintzileos WS, Vintzileos AM, Berghella V, et al. I. Adverse Pregnancy Outcomes Among Individuals With and Without Severe Acute Respiratory Syndrome Coronavirus 2 (SARS-CoV2): A Systematic Review and Meta-analysis. Obstet Gynecol. 2021. doi:10.1097/AOG.0000000000004320.

35. Ahlberg M, Neovius M, Saltvedt S, Söderling J, Pettersson K, Brandkvist C, et al. Association of SARS-CoV-2 Test Status and Pregnancy Outcomes. JAMA. 2021. doi:10.1001/jama.2020.19124.

36. Cherif A, Grobe N, Wang X, Kotanko P. Simulation of Pool Testing to Identify Patients With Coronavirus Disease 2019 Under Conditions of Limited Test Availability. JAMA Network Open. 2021.

doi:10.1001/jamanetworkopen.2020.13075.

37. Eberhardt JN, Breuckmann NP, Eberhardt CS. Challenges and issues of SARS-CoV-2 pool testing. Lancet Infect Dis. 2020. doi:10.1016/S1473-3099(20)30467-9.

38. Yelin I, Aharony N, Shaer-Tamar E, Argoetti A, Messer E, Berenbaum D, et al. Evaluation of COVID-19 RT-qPCR test in multi-sample pools. Clinical Infectious Diseases. 2020. Doi: https://doi.org/10.1093/cid/ciaa531.

\section{Figures}

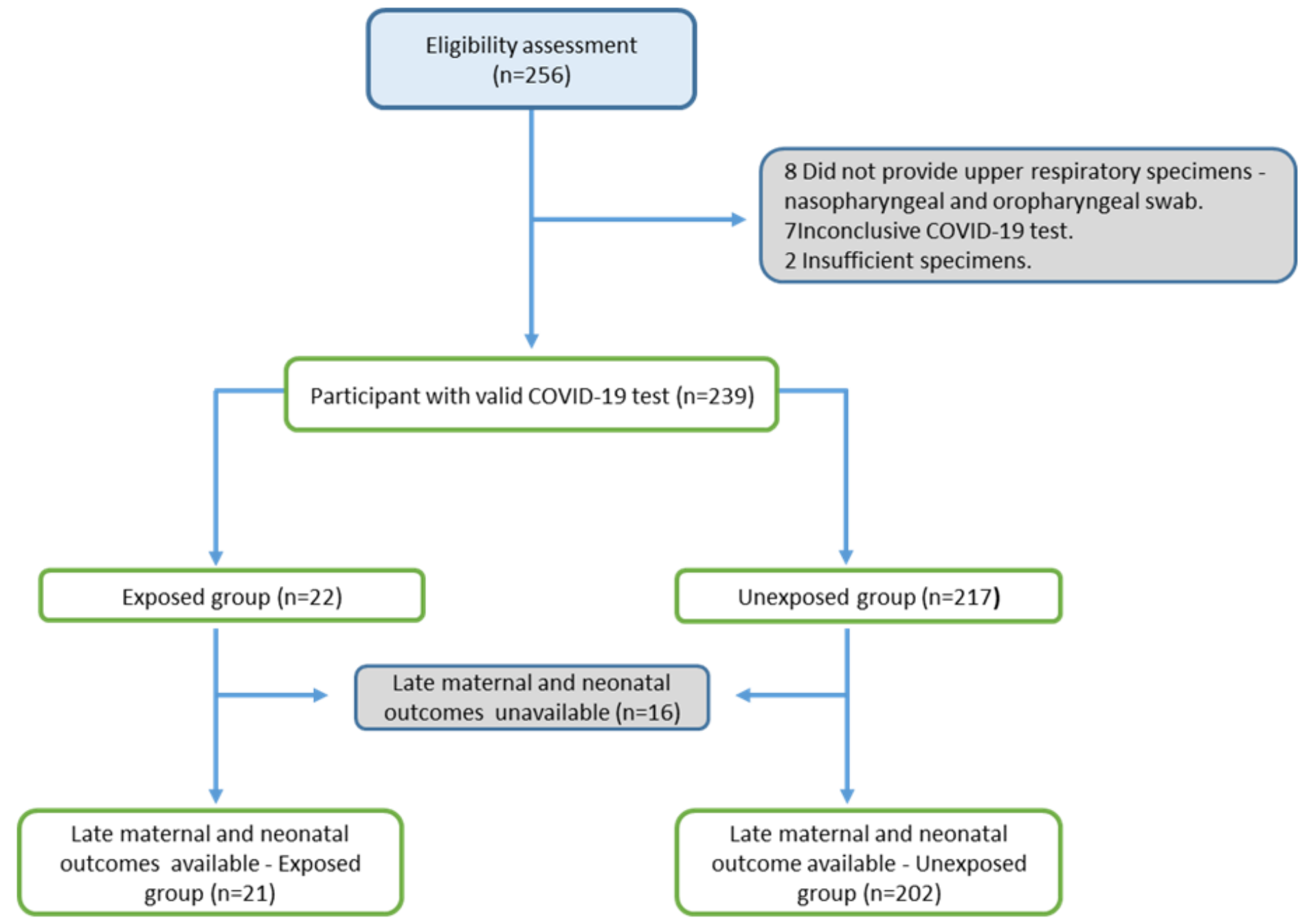

\section{Figure 1}

Study flowchart. 


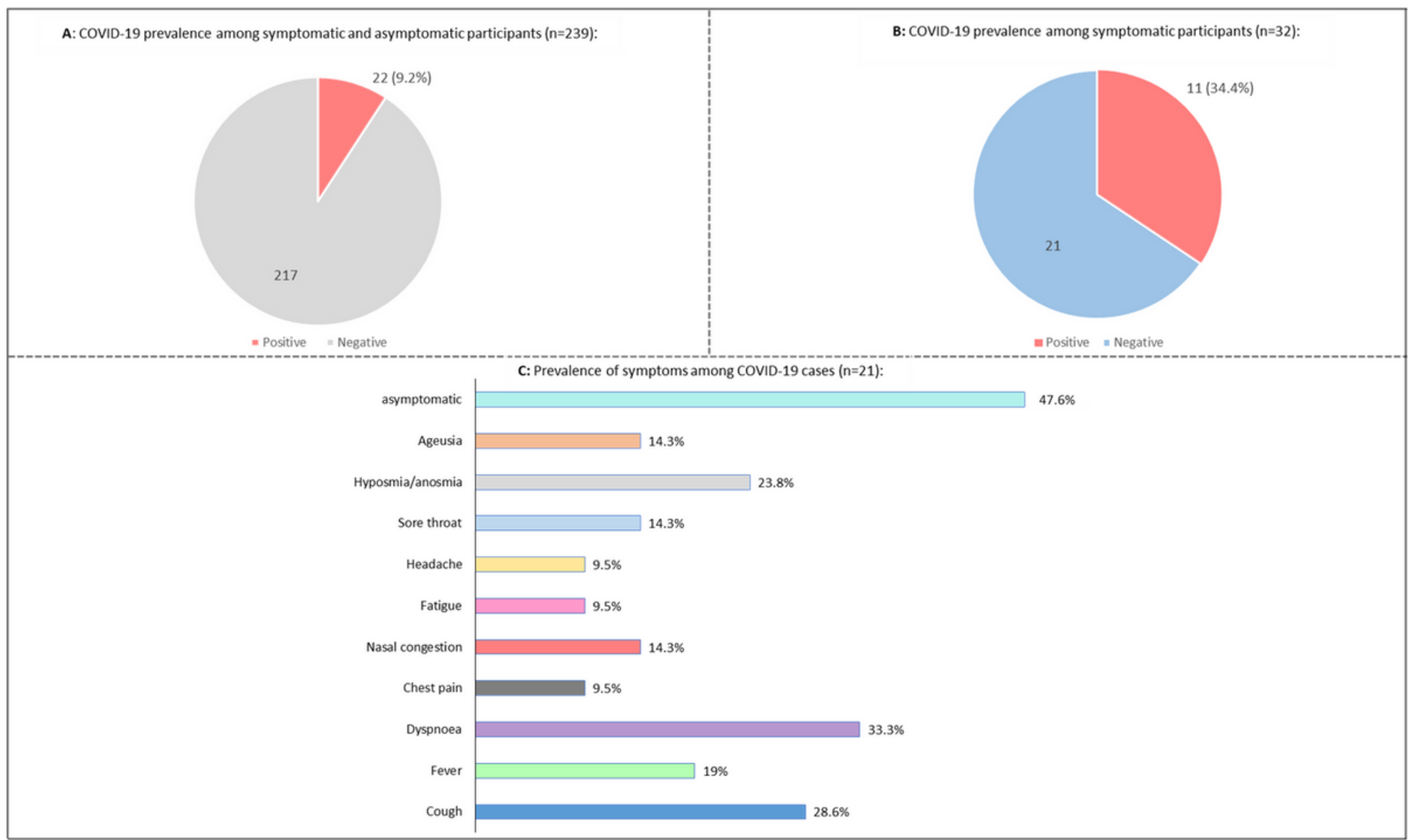

\section{Figure 2}

Prevalence of COVID-19 and symptoms among pregnant and postpartum women. 